\title{
Probing TeV scale physics in precision UCN decays
}

\section{Rajan Gupta*†}

Theoretical Division, Los Alamos National Laboratory, Los Alamos, NM 87545, USA

E-mail:rajan@lanl.gov

\section{Tanmoy Bhattacharya}

Theoretical Division, Los Alamos National Laboratory, Los Alamos, NM 87545, USA

E-mail:tanmoyalanl.gov

\section{Anosh Joseph}

Theoretical Division, Los Alamos National Laboratory, Los Alamos, NM 87545, USA

E-mail:anoshjoseph@gmail.com

\section{Saul D. Cohen}

Department of Physics, University of Washington, Seattle, WA 98195

E-mail:sdcohen@uw. edu

\section{Huey-Wen Lin}

Department of Physics, University of Washington, Seattle, WA 98195

E-mail:hwlinephys.washington. edu

\begin{abstract}
We present the calculation of matrix elements of iso-vector scalar, axial and tensor charges between a neutron and a proton state on dynamical $N_{f}=2+1+1$ HISQ configurations generated by the MILC Collaboration using valence clover fermions. These matrix elements are needed to probe novel scalar and tensor interactions in neutron beta-decay that can arise in extensions to the Standard Model at the TeV scale. Results are presented at one value of the lattice spacing, $a=0.12 \mathrm{fm}$, and two values of light quarks corresponding to $M_{\pi}=310$ and $220 \mathrm{MeV}$. We discuss two sources of systematic errors, contribution of excited states to these matrix elements and the renormalization constants, and the efficacy of methods used to control them.
\end{abstract}

31st International Symposium on Lattice Field Theory - LATTICE 2013

July 29 - August 3, 2013

Mainz, Germany

\footnotetext{
* Speaker.

${ }^{\dagger}$ LA-UR-13-29020
} 


\section{Introduction}

The standard model (SM) of elementary particles has been very successful in explaining phenomena up to the $\mathrm{TeV}$ scale and at the same time the observed electroweak symmetry breaking in the SM points to new physics at the $\mathrm{TeV}$ scale. In addition to the direct search for novel particles and interactions, one can also look for their effects in precision measurements at low energies. In Ref. [1], we showed that new scalar and tensor interactions at the $\mathrm{TeV}$ scale could give rise to corrections at the $10^{-3}$ level in precision measurements of the helicity flip parts of the decay distribution of (ultra)cold neutrons (UCN). This sensitivity is reachable in experiments currently under construction and being planned. Even if these experiments see a signal, to constrain the allowed parameter space of beyond the SM (BSM) models, however, requires that matrix elements of isovector scalar and tensor bilinear quark operators are known to 10-20\% accuracy. Lattice calculations are well poised to provide these estimates with the desired precision. In these proceedings, we summarize results on the charges $g_{A}, g_{S}$ and $g_{T}$ calculated on 2+1+1 flavor HISQ lattices [2] using clover valence quarks at one value of the lattice spacing, $a=0.12 \mathrm{fm}$, and two values of light quarks corresponding to $M_{\pi}=310$ and $200 \mathrm{MeV}$. Details are given in Ref. [3]. We will also discuss the efficacy of methods used to control two of the largest sources of systematic errors contribution of excited states and estimates of renormalization constants.

\section{Statistics}

The MILC Collaboration [2] has generated ensembles of roughly 5500 trajectories of 2+1+1flavor HISQ lattices at three values of light quark masses corresponding to $M_{\pi}=310,220,140$ $\mathrm{MeV}$ at $a=0.12,0.09$ and $0.06 \mathrm{fm}$. Here we focus on two ensembles of roughly 1000 configurations at $a=0.12 \mathrm{fm}$ with $M_{\pi}=305.3(4)$ and 216.9(2) MeV [2] (called $M_{\pi}=310$ and $220 \mathrm{MeV}$ ensembles). These are separated by 5 trajectories of the hybrid Monte Carlo evolution and five hundred trajectories are discarded for thermalization. On each configuration, we use four smeared sources, displaced both in time and space directions to reduce correlations. To evaluate the statistical significance of the data, we also analyze the data as two subsets with roughly 500 configurations. These two subsets give compatible results and the errors are roughly $\sqrt{2}$ larger compared to the full set. Our overall conclusions are: (i) the errors in $g_{S}$ are roughly five times those in $g_{A}$ and $g_{T}$ and (ii) while statistics of $O(1000)$ configurations provide estimates of $g_{S}$ with $15-20 \%$ uncertainty, to achieve this desired accuracy after chiral and/or continuum extrapoltions will require reducing the errors by at least another factor of two, i.e., increasing statistics by a factor of $4-9$.

\section{Excited-State Contamination}

The goal is to extract all observables (charges, charge radii, form factors) by calculating matrix elements between ground-state nucleons, however, nucleon operators used on the lattice couple to the ground state and all its radially excited states. The unwanted excited states contamination has to be removed to get the final estimate.

Assuming that only the leading excited state with mass $M_{1}$ and coupling $\mathscr{A}_{1}$ to our operator contributes significantly, we can write the three-point function with source at $t_{i}=0$, operator 

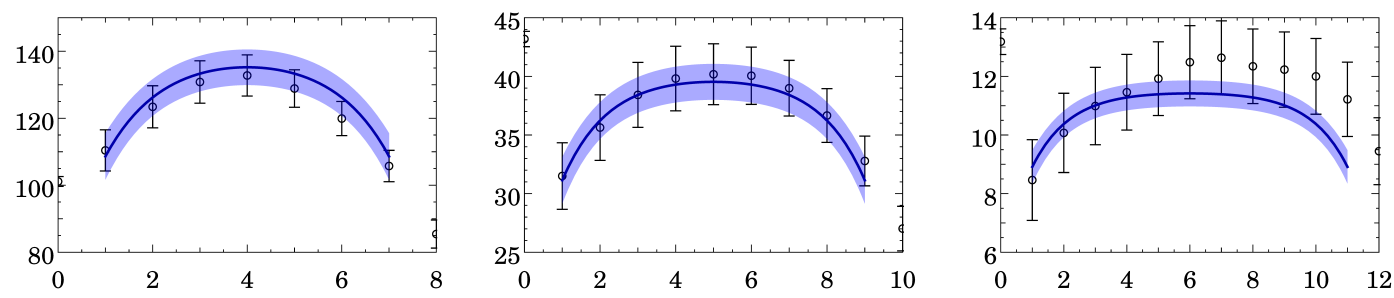

Figure 1: Fit using Eq.(3.1) to extract $g_{S}$ from the $220 \mathrm{MeV}$ pion ensemble data at $a=0.12 \mathrm{fm}$. A simultaneous fit is made to the three $t_{\text {sep }}=8,10$ and 12 data.

insertion at $t=t$ and sink at $t_{f}=t_{\mathrm{sep}}$ as

$$
\begin{aligned}
\mathscr{C}_{\Gamma}^{(3), T}\left(t_{i}, t, t_{f} ; \vec{p}_{i}, \vec{p}_{f}\right) & \approx\left|\mathscr{A}_{0}\right|^{2}\left\langle 0\left|O_{\Gamma}\right| 0\right\rangle e^{-M_{0}\left(t_{f}-t_{i}\right)}+\left|\mathscr{A}_{1}\right|^{2}\left\langle 1\left|O_{\Gamma}\right| 1\right\rangle e^{-M_{1}\left(t_{f}-t_{i}\right)} \\
& +\mathscr{A}_{0} \mathscr{A}_{1}^{*}\left\langle 0\left|O_{\Gamma}\right| 1\right\rangle e^{-M_{0}\left(t-t_{i}\right)} e^{-M_{1}\left(t_{f}-t\right)}+ \\
& +\mathscr{A}_{0}^{*} \mathscr{A}_{1}\left\langle 1\left|O_{\Gamma}\right| 0\right\rangle e^{-M_{1}\left(t-t_{i}\right)} e^{-M_{0}\left(t_{f}-t\right)}
\end{aligned}
$$

from which we need to extract $\left\langle 0\left|O_{\Gamma}\right| 0\right\rangle$. The masses and amplitudes $M_{0}, M_{1}, A_{0}$, and $A_{1}$ are obtained from the two-point functions. With these in hand, to extract $\left\langle 0\left|O_{\Gamma}\right| 0\right\rangle$ by isolating $\left\langle 0\left|O_{\Gamma}\right| 1\right\rangle$ and $\left\langle 1\left|O_{\Gamma}\right| 1\right\rangle$ requires that the calculations be done with multiple $t$ and $t_{\text {sep. }}$. We have carried out simulations at 5 values of $t_{\text {sep }}$ for the $M_{\pi}=310 \mathrm{MeV}$ ensemble and, based on insight gained from that analysis, on three values for the $M_{\pi}=220 \mathrm{MeV}$ ensemble. Using the sequential source method, operator insertion is carried out at all values of $t$ between the source and sink timeslices. We then apply a nonlinear least-square fitter that automatically selects a fit range within $t_{i}-t_{f}$ for each $t_{\text {sep }}$ value to reduce end effects and then fits data for all $t_{\text {sep }}$ simultaneously using Eq. (3.1).

Our analysis shows that excited state contamination, contributions of non-zero $\left\langle 0\left|O_{\Gamma}\right| 1\right\rangle$ and $\left\langle 1\left|O_{\Gamma}\right| 1\right\rangle$, is significant and can be eliminated by carrying out simulations at mutiple $t_{\text {sep }}$ and then doing a simultaneous fit to data at all $t_{\text {sep }}$ using Eq. (3.1). An example of the simultaneous fit to $t_{\text {sep }}=8,10,12$ data to extract $g_{S}$ using Eq.(3.1) for the $220 \mathrm{MeV}$ pion ensemble at $a=0.12 \mathrm{fm}$ is shown in Figure 1. We also show a comparison of estimates obtained from different fit procedures in Figure 2 and the efficacy of the 2-state simultaneous fit method. Highlights of our analysis for extracting the isovector charges $g_{A, S, T}$ are:

- The statistical errors increase by about $40 \%$ with each unit increase in $t_{\text {sep. }}$. This growth in errors limits the maximum $t_{\text {sep }}$ that can be analyzed reliably with given statistics.

- The 2-state simultaneous fit gives stable estimates of the central values and errors with respect to the range of $t$ selected for each $t_{\text {sep. }}$.

- For the nucleon operator used by us, estimates from a single state ansatz becomes consistent wth those from the 2-state simultaneous fit for $t_{\text {sep }}>1.2 \mathrm{fm}$.

- The signal in the extraction of $g_{S}$ is the noisiest and the errors are about five times those in $g_{A}$ and $g_{T}$. Nevertheless, on the $220-\mathrm{MeV}$ ensembles, the error estimate is about $15 \%$, reasonably close to our desired accuracy. 

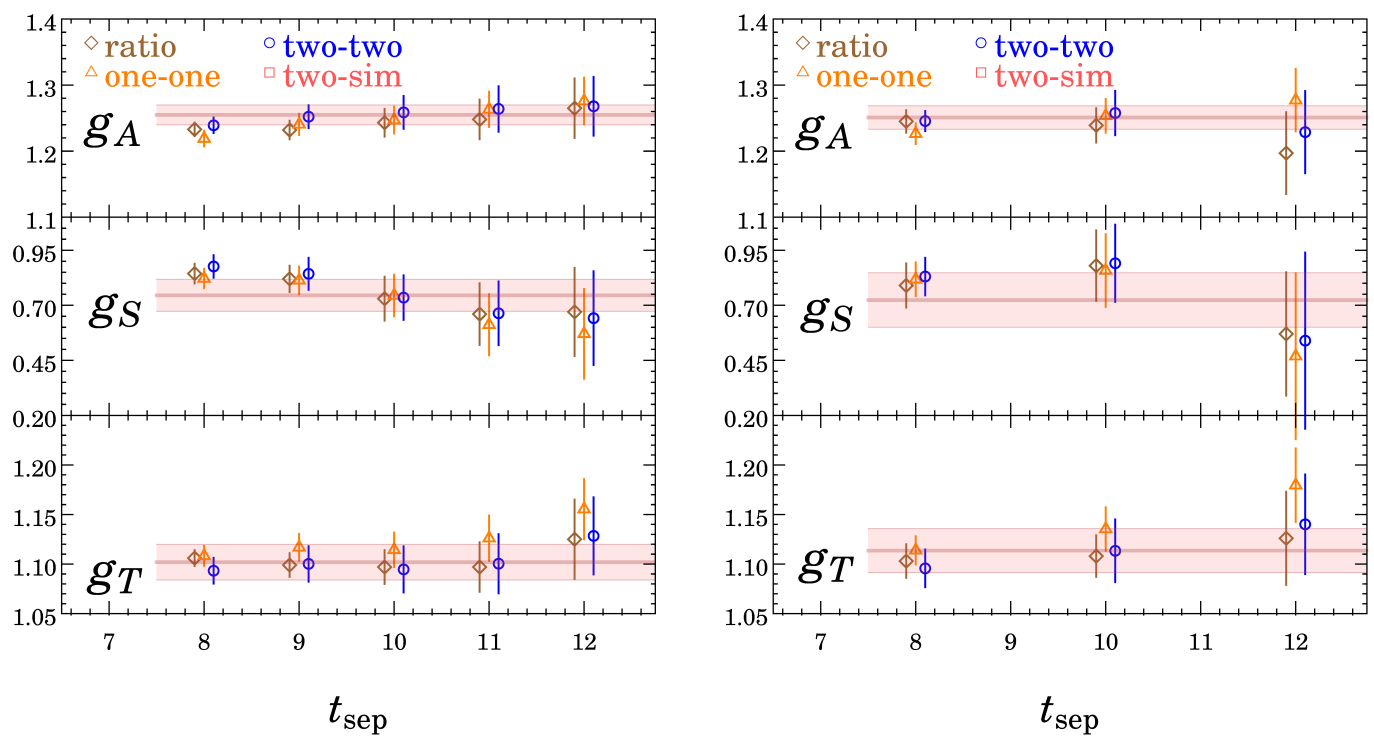

Figure 2: Comparison of estimates of the unrenormalized isovector charges $g_{A, S, T}$ as functions of $t_{\text {sep }}$ with $310 \mathrm{MeV}$ (left) and $220 \mathrm{MeV}$ (right) ensembles. The "ratio" and "one-one" method assumes a single state dominates; the "two-two" includes two states but analyzes data at each $t_{\text {sep }}$ separately. The band gives the result of the 2-state simultaneous fit to all $t_{\text {sep }}$ (two-sim).

- The errors increase by about $20 \%$ on lowering the light ( $u$ and $d$ ) quark masses by a factor of two, i.e., going from 310 to $220 \mathrm{MeV}$ ensemble. Unfortunately, the estimates at the two quark masses overlap within $1 \sigma$ errors, therefore a reliable chiral extrapolation cannot be made. Based on current data we conclude that the best strategy is to work directly at the physical light quark masses, especially since the theoretical analysis of the expected chiral behavior of these charges is not well-established.

We are in the process of performing the same analysis on $a=0.12,0.09$ and $0.06 \mathrm{fm}$ lattices at roughly the same light quark masses corresponding to pion masses of 310 and $220 \mathrm{MeV}$. There is significant improvement in the quality of the signal with decreasing lattice spacing. With data at multiple values of quark masses and lattice spacings in hand, we hope to elucidate the behavior versus quark masses and make the extrapolation to the continuum limit.

\section{Non-perturbative Renormalization}

We are using the RI-sMOM scheme to calculate the renormalization constants of the isovector bilinears non-perturbatively on the lattice [4]. This method relies on the presence of a window in momentum $q, \Lambda_{Q C D}<q<c / a$, where the lattice artifacts are small and $c$ is a number of $O(1)$ that is a priori unknown. Estimates in this window in the RI-sMOM scheme are matched to the $\overline{\mathrm{MS}}$ scheme at the same scale $q$ using 1-loop matching and then run to $2 \mathrm{GeV}$ using 2-loop expressions. Our results in both the RI-sMOM scheme and $\overline{\mathrm{MS}}$ scheme at $2 \mathrm{GeV}$ are shown in Figure 3 for $Z_{S}$ and $Z_{T}$ for the $310 \mathrm{MeV}$ pion ensemble at $a=0.12 \mathrm{fm}$. 

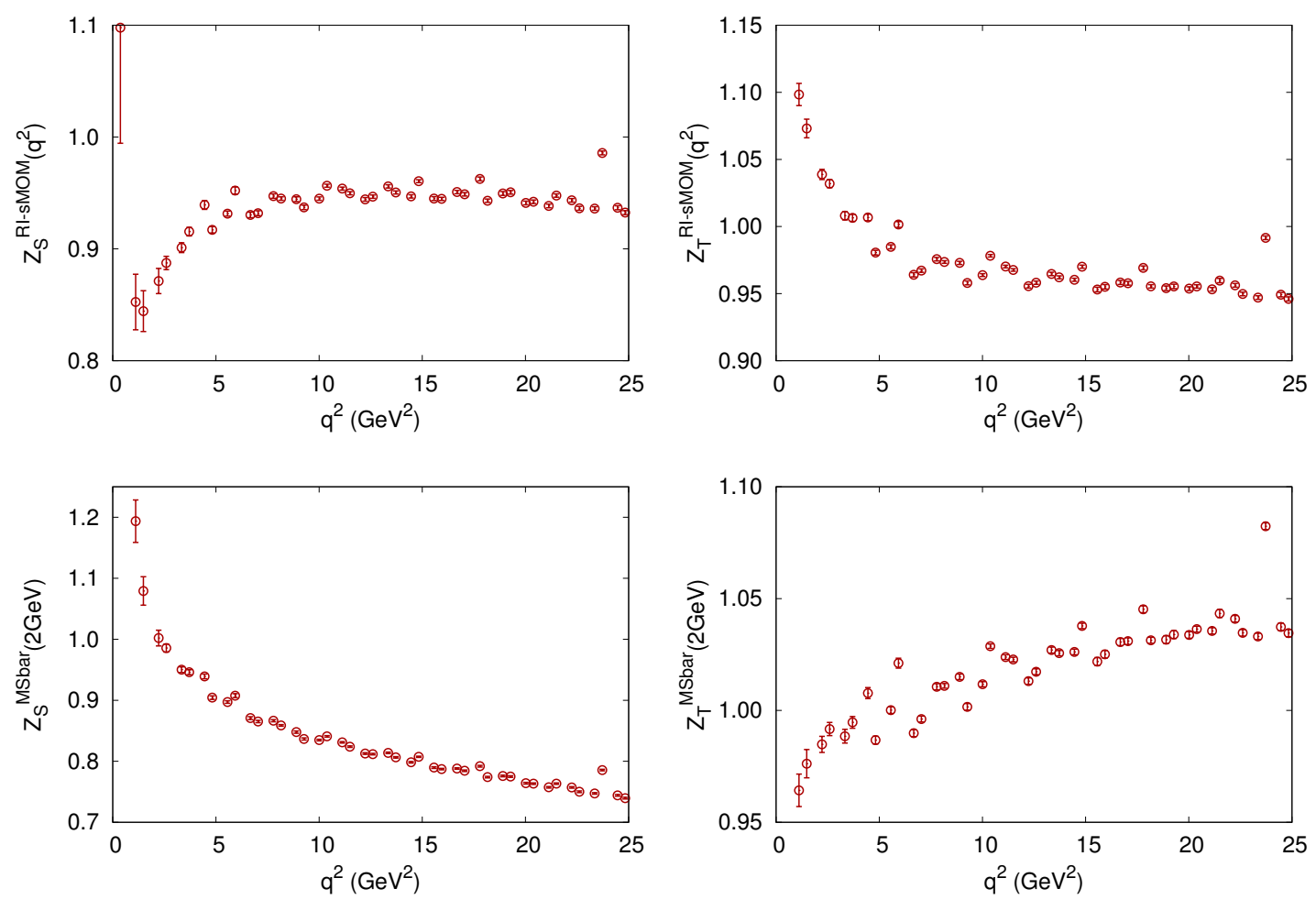

Figure 3: $Z_{S}$ and $Z_{T}$ in the RI-sMOM scheme (top) and after running to $2 \mathrm{GeV}$ in the $\overline{\mathrm{MS}}$ scheme (bottom) for the $310 \mathrm{MeV}$ ensemble at $a=0.12 \mathrm{fm}$. The $q^{2}$ is the lattice momentum in physical units and at each $q^{2}$ we have plotted the $Z$ with smallest $\sum q_{i}^{4} /\left(q^{2}\right)^{2}$,i.e., least breaking of rotational symmetry.

To interpret these results, it is important to establish the above mentioned window in $q^{2}$. Sufficiently close to the continuum limit, $Z_{S}$ and $Z_{T}$ in the RI-sMOM scheme should show a $q^{2}$ dependence given by the anomalous dimensions of these operators and a weaker dependence on the running of $\alpha_{s}$, i.e., this known $q^{2}$ dependence can be used to establish a scaling window. For values of $q^{2}$ in such a scaling window, the results after conversion to $\overline{\mathrm{MS}}$ scheme at $2 \mathrm{GeV}$ should be independent of $q^{2}$. It is not obvious from the data shown in Figure 3 that such a window exists in our $a=0.12 \mathrm{fm}$ data. Data suggest that the $a=0.12 \mathrm{fm}$ ensembles maybe too coarse to make contact with perturbation theory. We see a marked improvement on the $a=0.09$ and $0.06 \mathrm{fm}$ lattices.

A second possibility is that the artifacts introduced by HYP smearing of the lattice we use are large. Smearing is supposed to leave the long distance non-perturbative physics unchanged but smooth out the short distance fluctuations, i.e., it introduces artifacts at large $q^{2}$. Since smearing is a black box, it could shrink or completely obscure the scaling window for a given quantity as discussed in Refs. [5]. In such cases one could take the following approach. Assume that the calculation of the $Z^{\prime} s$ in RI-sMOM scheme has lattice artifacts that persist when converted to $\overline{\mathrm{MS}}$ scheme at $2 \mathrm{GeV}$. Then, for fixed $q^{2}$ and quark masses in physical units we could calculate say $Z_{S}(\overline{\mathrm{MS}}, 2 \mathrm{GeV}) g_{S}$ at different lattice spacings and extrapolate these estimates to the continuum limit. If all the significant systematics in the extrapolation to the continuum limit are well represented by extrapolation ansatz then in the continuum limit the results should be independent of $q^{2}$. These issues are currently being investigated using the data at all three lattice spacings. 


\section{Impacts of $g_{S, T}$ on Searches for New Physics}

Precision measurements of neutron (nuclear) beta-decay and deviations from well estimated standard model predictions would give hints of potential BSM physics at the TeV scale. We can analyze new physics in terms of an effective neutron beta-decay Hamiltonian:

$$
H_{\mathrm{eff}}=G_{F}\left(J_{V-A}^{\mathrm{lept}} \times J_{V-A}^{\mathrm{quark}}+\sum_{i} \varepsilon_{i}^{\mathrm{BSM}} \hat{O}_{i}^{\text {lept }} \times \hat{O}_{i}^{\text {quark }}\right),
$$

where $G_{F}$ is the Fermi constant, $J_{V-A}$ are the left-handed weak currents, and operators $\hat{O}_{i}$ have novel chiral structure. The low-energy couplings $\varepsilon$ encode both the fundamental couplings at the $\mathrm{TeV}$ scale and their evolution to the hadronic scale relevant to neutron decay. As discussed in Ref. [1], in neutron beta-decay only the isovector scalar and tensor operators contribute to $\hat{O}_{i}$.

Novel scalar and tensor interactions can also be probed at the LHC by analyzing the transverse mass distribution in the channel $p+p \rightarrow e^{-}+\bar{v}_{e}+X$. There, to distinguish novel from SM contributions, one has to look at the distribution much above the $W$ resonance [1]; the predicted bounds from LHC become tighter with increasing center-of-mass energy and integrated luminosity.

Figure 4 shows an illustrative comparison of the constraints on $\varepsilon_{S, T}$ (defined at $2 \mathrm{GeV}$ in the $\overline{\mathrm{MS}}$ scheme) obtained from both low-energy neutron decay and the CMS and ATLAS experiments at the LHC. We show three bounds from the LHC for different center-of-mass energies and integrated luminosity. To obtain these projected limits from the LHC, we use the tail of the transverse-mass distribution in the reaction $p p \rightarrow e \bar{v}+X$; that is, the region where $m_{T}>m_{T}^{\text {cut }}$. The transverse-mass cut is chosen such that the expected SM background is less than one event. For the brown ellipse, the background is taken from the measured value at CMS [6]; otherwise, the background is estimated by computing at tree level the transverse-mass distribution due to the production of a high- $p_{T}$ lepton from an off-shell $W$. For further details of this analysis, refer to Refs. [1,3] and [7]. The outer dashed purple ellipse gives the LHC expected constraint using the full current 8-TeV dataset; the inner dotted magenta ellipse gives the expected final LHC constraint with maximum lifetime luminosity at the $14-\mathrm{TeV}$ design energy.

We compare these LHC constraints to low-energy constraints using nuclear experiments. The outer blue region combines current nuclear experiments with model estimates of $g_{S, T}\left(0.25<g_{S}<\right.$ 1.0 and $\left.0.6<g_{T}<2.3[8]\right)$. The middle green region improves the constraint by using current lattice values for $g_{S, T}$. The inner red region combines nuclear experiment with anticipated future constraints from precision measurements of decays of ultracold neutrons (assuming $\left|b_{v}-b\right|<10^{-3}$ and $|b|<10^{-3}$ ) and future improvements in lattice values of $g_{S, T}$ to $10 \%$ uncertainty.

We find that the eventual reach of low-energy and LHC constraints are comparable. For the LHC, this requires the full integrated luminosity of $300 \mathrm{fb}^{-1}$ at $14-\mathrm{TeV}$ center-of-mass energy, whereas for low-energy probes it requires that UCN experiments attain bounds better than $10^{-3}$ and $g_{S, T}$ are calculated with better than $10 \%$ error.

\section{Acknowledgments}

We thank the MILC Collaboration for sharing the 2+1+1 HISQ lattices and Boram Yoon and Steve Sharpe for discussions. Simulations were performed using the Chroma software suite [9] on 


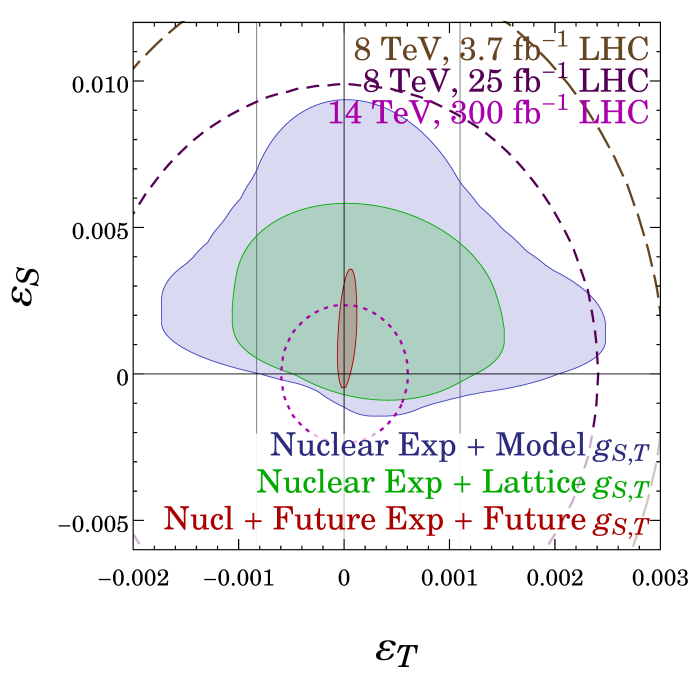

Figure 4: $\varepsilon_{S}-\varepsilon_{T}$ allowed parameter region using different experimental and theoretical inputs as discussed in the text. All estimates are in the $\overline{\mathrm{MS}}$ scheme at $2 \mathrm{GeV}$.

facilities of the USQCD Collaboration funded by the U.S. DoE and Extreme Science and Engineering Discovery Environment (XSEDE) supported by NSF grant number OCI-1053575. RG is supported by DOE grant DE-KA-1401020 and the LDRD program at LANL.

\section{References}

[1] T. Bhattacharya, etal.,Probing Novel Scalar and Tensor Interactions from (Ultra)Cold Neutrons to the LHC, Phys.Rev. D85 (2012) 054512.

[2] Bazavov, A., et al., MILC Collaboration, Phys. Rev D87 (2013) 054505.

[3] T. Bhattacharya, etal., Nucleon Charges and Electromagnetic Form Factors from 2+1+1-Flavor Lattice QCD, arXiv:hep-lat/1306.5435.

[4] G. Martinelli, et al., A General method for nonperturbative renormalization of lattice operators, Nucl.Phys. B445 (1995) 81. C. Sturm, et al., Renormalization of quark bilinear operators in a momentum-subtraction scheme, Phys.Rev. D80 (2009) 014501.

[5] R. Arthur, et al., A note on Rome-Southampton Renormalization with Smeared Gauge Fields, arXiv:1306.0835. A. T. Lytle and S. Sharpe, Non-perturbative Renormalization for Improved Staggered Bilinears, Phys. Rev. D88 054506.

[6] CMS Report, Search for leptonic decays of W' bosons in pp collisions at sqrt(s)=8 TeV, CMS-PAS-EXO-12-010, CERN, Geneva, 2012.

[7] V. Cirigliano, et al., Non-standard Charged Current Interactions, JHEP 1302 (2013) 046.

[8] P. Herczeg, Beta decay beyond the standard model, Prog. Part. Nucl. Phys. 46 (2001) 413.

[9] R. Edwards, B. Joo, Chroma Software System for LQCD, Nucl. Phys. Proc. Suppl. 140 (2005) 832 